\title{
PRODUCCIÓN INDEPENDIENTE DE TELEVISIÓN EN ANDALUCÍA. APROXIMACIÓN A LA ESTRUCTURA DE UN SECTOR DESCONOCIDO
}

\author{
David Fernández-Quijada21 \\ (Universitat Autònoma de Barcelona) \\ david.fernandez@uab.es
}

\begin{abstract}
Resumen: El crecimiento del sector de la producción independiente para televisión lo sitúa como un segmento cada vez más importante dentro de la industria audiovisual. Este artículo plantea un estudio de la producción independiente en Andalucía, situando su peso en relación con el conjunto de España, caracterizando y clasificando a sus empresas y analizando el rol jugado por la televisión pública autonómica para el desarrollo de esta industria.
\end{abstract}

Palabras clave: Producción independiente, industria audiovisual, Andalucía, Canal Sur, análisis de redes sociales.

Abstract: The independent TV production sector is becoming increasingly important for the audiovisual sector thanks to its growth. This article studies the independent production in Andalusia, measures it in relation with its Spanish counterparts, characterizes and classifies its firms and analyses the role played by the autonomous public service broadcaster in its development.

Keywords: Independent production, audiovisual industry, Andalusia, Canal Sur, social network analysis.

\section{INTRODUCCIÓN}

I sector audiovisual se ha convertido en los últimos años en uno de los que mejor ejemplifican el avance hacia la denominada Sociedad de la Información. Más allá de la realidad creciente del propio sector, las promesas futuras y los discursos políticos han coadyuvado a la visión del audiovisual como un sector estratégico en este contexto.

\footnotetext{
${ }^{21}$ El autor desea agradecer a Luis Arboledas (Universidad de Granada), Marina Ramos y María del Mar Ramírez (ambas de la Universidad de Sevilla) sus sugerencias a una versión previa de este texto. Todos los errores u omisiones son responsabilidad exclusiva del autor.
} 
Producción independiente de televisión en Andalucía. Aproximación a la estructura de un sector desconocido

Frecuentemente, además, se ha dado como absoluto el binomio que une el audiovisual con el desarrollo territorial, ignorando que el contexto actual de las industrias culturales se caracteriza por un marco de centralización de sus actividades a nivel global y estatal (Zallo, 2002). En consecuencia, previamente a los discursos resulta necesario conocer cuál es la realidad del sector, de manera que de su diagnóstico puedan adaptarse las estrategias empresariales y las políticas públicas adecuadas para el cumplimiento de las funciones sociales que tiene atribuidas y para el desarrollo del mismo, que ya se están llevando a cabo, tanto en Andalucía como en otras comunidades autónomas (Casado, 2005).

Para comprender la importancia del sector audiovisual sólo hace falta fijarse en el Directorio Central de Empresas (DIRCE) ${ }^{22}$, en el que existen dos categorías dentro de las que típicamente se encuadran las compañías del sector audiovisual: actividades cinematográficas y de vídeo (código CNAE-93 921) y actividades de radio y televisión (código CNAE-93 922) ${ }^{23}$. Si se observan los datos referentes a Andalucía se puede comprobar un alza sostenida en el número de empresas en ambas actividades. Entre 1999 y 2006, el crecimiento en actividades cinematográficas y de vídeo fue del $68,87 \%$ (casi un $10 \%$ anual), pasando de 379 a 640 compañías (gráfico 1). En el segmento de actividades de radio y televisión, el crecimiento fue del $79,15 \%$ (más del $11 \%$ anual), pasando de 235 a 421 sociedades. Entre ambas categorías se sobrepasó la cifra del millar de empresas en el año 2006.

Gráfico 1. Número de empresas audiovisuales andaluzas.

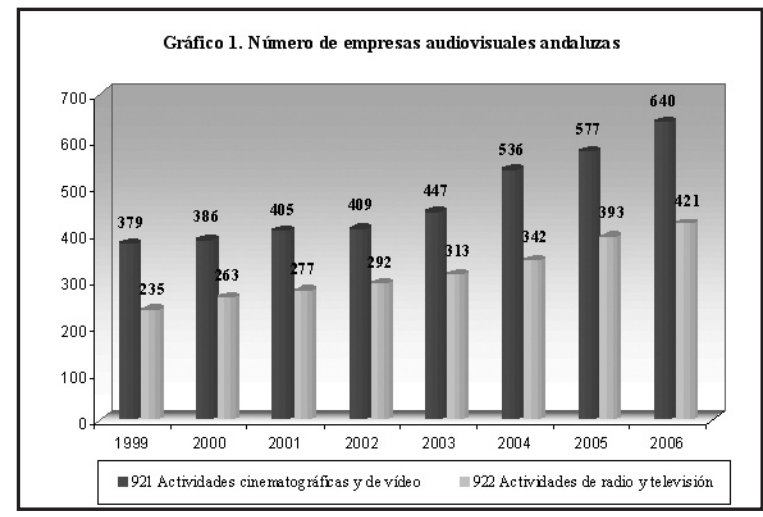

Fuente: Elaboración propia a partir de DIRCE.

\footnotetext{
${ }^{22}$ EI DIRCE es un servicio del Instituto Nacional de Estadística (INE) que clasifica a las sociedades mercantiles en función de su actividad.

${ }^{23}$ El Código Nacional de Actividades Económicas (CNAE) permite la comparación estadística en función de las actividades económicas de las empresas. Este código tiene algunos inconvenientes, ya que las actividades que declaran las empresas no siempre se corresponden con su actividad real o ésta puede comprender diversas actividades y, por tanto, entrar en diferentes categorías CNAE que no siempre son especificadas. En cualquier caso, a grandes rasgos su valor es indicativo de esa actividad.
} 
Estos datos ayudan a ubicar la magnitud del sector audiovisual, heterogéneo por naturaleza y que comprende actividades diversas como la producción, distribución y comercialización de productos televisivos, videográficos, radiofónicos o cinematográficos, entre otros, así como la gestión de derechos.

A lo largo de las siguientes páginas se presentan los resultados de una investigación que pretendía realizar una primera aproximación exploratoria a la estructura de uno de los segmentos clásicos de este sector, el de la producción televisiva. Se trata de un área emergente de la que apenas se ha ocupado la investigación académica, lo que ha creado un vacío cuyo abordaje parece necesario para entender el cada día más complejo sector televisivo.

\section{METODOLOGÍA}

La metodología aplicada partía de la explotación de los datos referidos a la producción independiente en las televisiones españolas de ámbito estatal y autonómico que anualmente publica el Gabinete de Estudios de la Comunicación Audiovisual (GECA) en su anuario. Concretamente, se utilizaron los datos referidos a las temporadas 2004/05 (GECA, 2006) y 2005/06 (GECA, 2007) ${ }^{24}$, tratándose este último de un momento importante ya que a inicios de la segunda de estas temporadas se produjo el cambio de modelo de la televisión de Sogecable desde el codificado Canal+ a un modelo abierto bajo la marca Cuatro, además de la aparición en escena de un nuevo canal hertziano en abierto, La Sexta.

Con estos datos se procedió a la identificación de las productoras, una tarea no siempre fácil por las diferencias entre las marcas comerciales de las mismas y sus denominaciones fiscales. Una vez realizada la identificación, accedimos a la información disponible en el Registro Mercantil ${ }^{25}$ sobre dichas empresas a través de los informes preceptivos que éstas deben depositar anualmente ${ }^{26}$.

Una parte de la información obtenida en el Registro Mercantil permitió localizar geográficamente a las empresas y distribuirlas por comunidades autónomas y provincias. De esta manera, se pudo determinar el volumen de producción a partir de estas unidades territoriales. Además, la naturaleza relacional de los datos de GECA permitió aplicar un análisis de redes sociales (ARS) a los diferentes actores identificados: no solamente se determinan las características de los dife-

\footnotetext{
${ }^{24}$ Los datos publicados por GECA proceden de las mediciones de Taylor Nelson Sofres y de las propias productoras y se refieren a la programación entre las $06: 30 \mathrm{~h}$ de la mañana y las 02:30h del día siguiente, es decir, un total de 20 horas diarias. En esta clasificación se incluyen programas contenedores y se excluyen aquéllos de duración inferior a quince minutos.

${ }^{25}$ El acceso al Registro Mercantil se produjo de manera electrónica a través del Sistema de Análisis de Balances Ibéricos (SABI) de la empresa Bureau van Dijk. Los datos están actualizados con la información disponible a fecha de 14 de marzo de 2009.

${ }^{26} \mathrm{~A}$ pesar de esta obligatoriedad, las empresas no siempre la cumplen en los plazos establecidos al respecto, de manera que se dan algunas lagunas estadísticas.
} 
Producción independiente de televisión en Andalucía.

Aproximación a la estructura de un sector desconocido

rentes actores sino que se analizan las relaciones entre ellos, estableciendo sus vínculos y sus patrones de relación. Para ello se procedió, tal como se indica en Fernández Quijada (2008). Primero se construyó una matriz rectangular de modo 2 de productoras independientes y televisiones y una segunda matriz atributiva complementaria de la primera en la que se introdujeron las variables para, posteriormente, representar gráficamente estas relaciones a través de sociogramas. La herramienta informática empleada fue UCINET 6 (Borgatti, Everett, Freeman, 2002), utilizando NetDraw para elaborar los sociogramas con los que se representan gráficamente estos datos.

\section{LA LÓGICA DE LA PRODUCCIÓN INDEPENDIENTE}

La producción audiovisual se inserta en el marco de las industrias culturales, dentro de cuya lógica se integra. A menudo esta producción se presenta junto a otras actividades de la cadena de valor, como la difusión, o se puede producir autónomamente y ser vendida en un mercado a los clientes potenciales, en este caso los radiodifusores. Es decir, la clásica dicotomía a la que se enfrentan los radiodifusores entre producir internamente o comprar a un proveedor externo.

El presente artículo se centra en el segmento de la producción para televisión, que comprende tanto la denominada producción financiada, realizada por un productor independiente, normalmente con los recursos de la cadena y financiada totalmente por ésta, como la producción asociada, en la que la cadena participa con productores independientes nacionales (Bustamante, 1999).

Sobre este tipo de producción se ha construido en los últimos años en España todo un sector industrial de compañías de producción denominadas independientes, aunque su grado real de dependencia es variable, ya que en la mayoría de ocasiones utilizan los equipos técnicos e incluso humanos de las propias televisiones que con posterioridad emitirán los productos audiovisuales. Además, algunas de estas productoras han sido adquiridas o creadas por grupos de comunicación con intereses en la difusión televisiva e, incluso, algunas de las productoras más potentes han emprendido el camino de la integración vertical descendente y han entrado en el accionariado de cadenas televisivas. En cualquier caso, a efectos nominales en este texto se consideran tales compañías como independientes ya que la producción de programas se realiza por parte de una empresa ajena a la que los emite, aunque puedan tener vínculos accionariales entre ellas.

\section{UNA CARACTERIZACIÓN DE LA PRODUCCIÓN INDEPENDIENTE EN AN- DALUCÍA}

Un primer dato necesario para mapear el sector de la producción independiente es el volumen de horas emitidas por las diferentes televisiones. En la temporada 2004/05, la producción independiente andaluza sumó $2.247,65$ horas, lo que representó un $12,46 \%$ de las $18.043,11$ horas de producción independiente 
contabilizadas en toda España. En la siguiente temporada, la producción de esta comunidad se quedó en $2.180,77$ horas, el $11,21 \%$ de las $19.455,42$ horas producidas en todo el estado. Así, mientras la demanda de producción independiente en España creció un 7,83\% (en gran parte, aunque no exclusivamente, por la aparición de Cuatro y La Sexta), en Andalucía disminuyó un 2,97\%. Estos datos, además, se sitúan ligeramente por debajo del peso que correspondería a esta comunidad autónoma en función de su producto interior bruto (PIB), situado en 2005 en el $13,82 \%$ y en el $13,86 \%$ un año después ${ }^{27}$.

A pesar de esta ligera regresión, los datos generales históricos resultan más optimistas. Si hace casi una década una investigación señalaba que sólo existían cuatro empresas andaluzas con capacidad para elaborar un producto audiovisual completo (Fernández Soriano, 1999), en la temporada 2004/05 hasta 23 productoras de la comunidad figuraban en la lista de proveedores de las cadenas de ámbito estatal y autonómico, una cifra que en la siguiente temporada había bajado hasta $21^{28}$. En la segunda de estas temporadas aparecían además dos nuevos actores ausentes de la primera. Así, la tabla 1 muestra la relación de productoras localizadas en Andalucía, su volumen de producción y el porcentaje de mercado a nivel autonómico que conseguían.

\footnotetext{
${ }^{27}$ Datos recogidos de la serie Contabilidad Regional de España de la web del INE.

${ }^{28}$ Este dato no significa que esta veintena de productoras sean capaces de elaborar un producto audiovisual completo de manera orgánica, como se deduce de la investigación de Fernández Soriano, pero resulta relevante por cuanto hace referencia a la evolución de la industria audiovisual localizada en la región.
} 
Producción independiente de televisión en Andalucía. Aproximación a la estructura de un sector desconocido

Tabla 1. Productoras andaluzas por cuota de mercado.

\begin{tabular}{|c|c|c|c|c|c|c|c|c|}
\hline \multicolumn{5}{|c|}{$2004 / 05$} & \multicolumn{4}{|c|}{$2005 / 06$} \\
\hline Productoras & $\mathbf{N}^{0}$ & Horas & $\%$ & & Productoras & $\mathrm{N}^{0}$ & Horas & $\%$ \\
\hline Producciones 52 & 3 & 632,65 & 28,15 & 1 & Producciones 52 & 2 & 630,18 & 28,90 \\
\hline Atrium Digital & 1 & 509,12 & 22,65 & 2 & Atrium Digital & 1 & 549,55 & 25,20 \\
\hline ZZJ & 4 & 270,78 & 12,05 & 3 & ZZJ & 9 & 290,07 & 13,30 \\
\hline Caligari Films & 1 & 114,97 & 5,11 & 4 & Caligari Films & 4 & 159,38 & 7,31 \\
\hline Mettre & 4 & 113,17 & 5,03 & 5 & ADM & 1 & 116,78 & 5,36 \\
\hline Estarciera & 1 & 112,35 & 5,00 & 6 & Linze & 1 & 106,75 & 4,90 \\
\hline Linze & 2 & 110,45 & 4,91 & 7 & Mettre & 3 & 70,42 & 3,23 \\
\hline Tarea Sur & 1 & 63,22 & 2,81 & 8 & Silencio & 1 & 47,13 & 2,16 \\
\hline REDacción 7 And. & 2 & 61,20 & 2,72 & 9 & REDacción 7 And. & 1 & 32,05 & 1,47 \\
\hline ADM & 1 & 49,97 & 2,22 & 10 & Tarea Sur & 2 & 26,63 & 1,22 \\
\hline Silencio & 1 & 48,43 & 2,15 & 11 & Lula & 1 & 25,87 & 1,19 \\
\hline Jondo & 1 & 29,87 & 1,33 & 12 & Videoplaning & 2 & 25,85 & 1,19 \\
\hline Media Sur & 2 & 22,35 & 0,99 & 13 & Al Andalus & 1 & 23,35 & 1,07 \\
\hline Al Andalus & 1 & 22,25 & 0,99 & 14 & Media Sur & 1 & 12,47 & 0,57 \\
\hline Lula & 1 & 19,12 & 0,85 & 15 & Digit Y Suit & 1 & 12,23 & 0,56 \\
\hline División XL & 1 & 17,73 & 0,79 & 16 & División XL & 1 & 11,97 & 0,55 \\
\hline PC 29 & 1 & 15,28 & 0,68 & 17 & Savitel & 1 & 11,43 & 0,52 \\
\hline Savitel & 4 & 15,13 & 0,67 & 18 & PC 29 & 1 & 11,13 & 0,51 \\
\hline Digit Y Suit & 1 & 8,37 & 0,37 & 19 & Euro Programmes & 1 & 9,28 & 0,43 \\
\hline Videoplaning & 2 & 6,05 & 0,27 & 20 & Diputación de Cádiz & 1 & 4,95 & 0,23 \\
\hline SANDETEL & 1 & 1,77 & 0,08 & 21 & Estarciera & 1 & 3,28 & 0,15 \\
\hline Imago & 1 & 1,72 & 0,08 & 22 & & & & \\
\hline Zig Zag & 1 & 1,72 & 0,08 & 23 & & & & \\
\hline TOTAL & 38 & $2.247,65$ & 100 & & TOTAL & 37 & $2.180,77$ & 100 \\
\hline
\end{tabular}

Fuente: Elaboración propia a partir de GECA y SABI.

La situación de los 25 actores identificados era bastante estable y, de hecho, las primeras cuatro empresas (Producciones 52, Atrium Digital, ZZJ y Caligari Films) repetían el cuadro de liderazgo regional por volumen de horas en ambas temporadas. Además, se observaba un alto grado de concentración de las horas producidas en un pequeño número de empresas. En las dos temporadas el mercado estaba encabezado por Producciones 52, con cuotas de mercado superiores al $28 \%$. Las tres primeras empresas sumaban una cuota del $62,85 \%$ en la temporada 2004/05, un porcentaje que ascendía hasta el $67,40 \%$ un año después. Los cinco primeros actores, por su parte, sumaban el $72,99 \%$ de cuota 
en 2004/05, para elevarse al $80,07 \%$ doce meses después. El grado de concentración, ya importante en la primera temporada analizada, subía, pues, de manera destacada en 2005/06. En 2004/05, la empresa andaluza con mayor volumen de producción, Producciones 52, se situaba novena dentro del ranking español, en cuyas veinte primeras posiciones también aparecían Atrium Digital y ZZJ. Para la temporada 2005/06, las tres escalaban posiciones: Producciones 52 ascendía un puesto hasta el octavo y Atrium Digital y ZZJ, dos cada una para situarse undécima y decimoséptima respectivamente (Fernández-Quijada, 2009).

Si la clasificación se efectúa por número de producciones, una variable mucho menos fiel por la diversidad de los programas en duración, periodicidad y longevidad, en 2004/05 destacaban ZZJ, Mettre y Savitel, con cuatro programas cada una, mientras que en 2005/06 ZZJ concentraba casi una cuarta parte de los programas producidos en Andalucía, con un total de nueve. Entre ambas temporadas el número de programas experimentó un ligero retroceso (de 38 a 37), con algunos de ellos disponibles en más de una televisión: en 2004/05, "Moranquísimo" de Estarciera se difundía por Canal Sur pero también por las cadenas autonómicas de Castilla-La Mancha y las Islas Canarias, y "Loco mundo loco" de Tarea Sur repetía en Canal Sur y Castilla-La Mancha Televisión. En 2005/06, "Menuda noche" de ZZJ se podía ver a través de Canal Sur y el Canal 9 valenciano, mientras que hacían doblete en los dos canales andaluces "Retratos" de PC 29 y "Canal Andalucía Turismo" de ADM.

Un dato relevante del análisis de estas empresas era la pertenencia de dos de ellas a grandes grupos de comunicación. En primer lugar, Linze, la única con sede en Granada, filial del conglomerado holandés Endemol, que también operaba en España con dos de las productoras más grandes del país, Gestmusic-Endemol y Zeppelin Televisión, además de Diagonal Televisió. Linze era la productora de "Arrayán" y "La noche de Arrayán" para Canal Sur. En segundo término, el grupo Mediapro tenía presencia a través de su filial Media Sur, que produjo "Almería olímpica" en 2004/05 y "Bienvenidos" en las dos temporadas, ambas para Canal Sur. Ello no era óbice para observar también en Andalucía la presencia directa de su matriz, tal como se verá al analizar a los proveedores de Canal Sur.

Aún así, en el conjunto del sector andaluz estas empresas eran poco representativas, destacando la presencia de las productoras realmente independientes respecto de los grupos de comunicación, algunas de ellas ligadas a personajes del mundo del periodismo y el espectáculo, como Jesús Quintero (Silencio) o el dúo cómico Los Morancos (Estarciera). Una de ellas, REDacción 7 Andalucía, estaba controlada por la productora madrileña REDacción 7 , cuyo director ejecutivo y administrador único era el periodista andaluz Paco Lobatón, primera imagen que apareció en Canal Sur y primer director de sus servicios informativos (Castro, Manfredi, Navarro, 1999). Igualmente, en 2004/05 era el presentador de "Siete lunas" y, en la temporada 2005/06, de "La vida en tiempo real". A pesar de que las marcas de los programas que producía para Canal Sur estaban registradas 
Producción independiente de televisión en Andalucía. Aproximación a la estructura de un sector desconocido

por la sociedad matriz, la mayor parte de la producción se realizaba en las instalaciones de la filial sevillana ${ }^{29}$.

En el caso andaluz se daba una particularidad respecto a las productoras de otras comunidades autónomas, que era la presencia del sector público dentro de este conjunto de empresas. Lo hacía a través de SANDETEL (Sociedad Andaluza para el Desarrollo de las Telecomunicaciones S.A.), controlada por la Agencia de Innovación y Desarrollo de Andalucía, un organismo dependiente de la Consejería de Innovación, Ciencia y Empresa de la Junta de Andalucía. En SANDETEL, además, participaba la Radio Televisión de Andalucía (RTVA) a través del 49\% que poseía de las acciones. A la vez, SANDETEL era el accionista mayoritario de ADM (Andalucía Digital Multimedia), en la que participaba junto a diversas cajas de ahorro andaluzas, la Universidad de Málaga y el operador de telecomunicaciones Axión. En el accionariado de Axión, controlado por France Télécom, estaba también presente SANDETEL con un $23,4 \%$ de las acciones, ya que esta sociedad era la mayoritaria dentro de Red de Banda Ancha de Andalucía, sociedad que fusionada con Medialatina dio origen a Axión. Igualmente, SANDETEL figuraba como socio minoritario de la productora ZZJ en la empresa de subtitulado Mundovisión MGI 2000. También la Sociedad Estatal de Participaciones Industriales (SEPI) había invertido más de siete millones y medio de euros en Videoplaning a través de SEPI Desarrollo Empresarial. Finalmente, en la temporada 2005/06 aparecía en la lista de productoras la Diputación de Cádiz, que figuraba como responsable de "El mar de la libertad" para Canal Sur.

Por lo que se refiere a la localización de todo este tejido productivo, en 2004/05 el $91,02 \%$ del volumen de producción fue responsabilidad de compañías ubicadas en la provincia de Sevilla (gráfico 2). Es muy reseñable el detalle de que más de dos terceras partes $(67,39 \%)$ de la producción andaluza se realizaron en la localidad de Mairena del Aljarafe, una población de apenas 39.065 habitantes, un $0,5 \%$ del total de la región. En 2005/06 los datos eran bastante similares, con Sevilla acaparando un $87,27 \%$ de la producción, con un papel central para las productoras de Mairena del Aljarafe, que en conjunto sumaban el $61,86 \%$ de la producción andaluza.

\footnotetext{
${ }^{29}$ Según María José Villalobos, directora de producción de REDacción 7 Andalucía, los equipos de algunos de estos espacios estaban divididos entre Madrid y Sevilla. A la vez, la filial sevillana también participaba en producciones para cadenas de televisión de otras regiones, aunque en esos casos se ha optado por asignar el conjunto de la producción a la sociedad matriz (comunicación telefónica personal con el autor, 28 de abril de 2008).
} 
Gráfico 2. Volumen de producción independiente andaluza por provincias (\%).

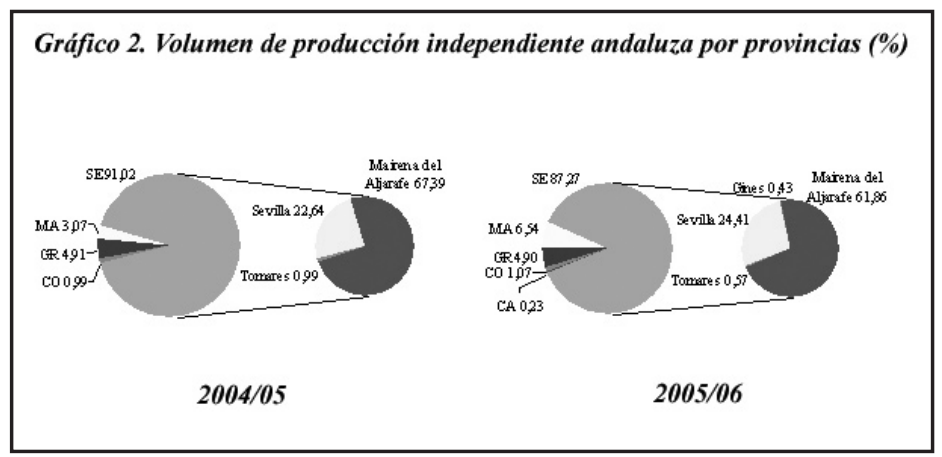

Fuente: Elaboración propia a partir de GECA y SABI.

En esta localidad de la primera corona metropolitana sevillana se ubicaban 9 de las 25 productoras identificadas, ocho de las cuales se concentraban en el Parque Industrial y de Servicios del Aljarafe (PISA). Concretamente, en este enclave tenían su sede las dos primeras productoras andaluzas, Producciones 52 y Atrium Digital, así como Mettre, Tarea Sur, REDacción 7 Andalucía, PC 29, Videoplaning e Imago, a las que había que sumar una cincuentena más de empresas relacionadas con el sector audiovisual y publicitario. La novena productora de la ciudad era Estarciera. En el resto de la comarca del Aljarafe también se ubicaban otras productoras como Media Sur (en Tomares) o Euro Programmes (en Gines), además de la propia sede de Canal Sur Televisión en el municipio colindante de San Juan de Aznalfarache. Si a ello sumamos la cercana capital andaluza y sus nueve productoras (ZZJ, SANDETEL, Silencio, Caligari Films, Jondo, Zigzag, Savitel, División XL y Digit Y Suit -esta última con instalaciones en el polígono PISA y con la sede social trasladada a Bormujos, también en la comarca del Aljarafe, tras las temporadas analizadas-), encontramos un reducido espacio alrededor de Sevilla en el que se concentraba el núcleo central del tejido industrial del audiovisual andaluz. El resto de localidades en las que se ubicaban las productoras era Málaga (ADM y Lula), Córdoba (Al Andalus), Cádiz a través de su Diputación y Granada, en la que radicaban las oficinas centrales de Linze, cuya producción estrella, "Arrayán", se producía íntegramente en las instalaciones de Loasur Audiovisual en el municipio malagueño de Coín (Martínez, 2007).

Más allá del volumen de producción original programada por las diferentes televisiones, el criterio de la facturación también puede ayudar a obtener una visión de la situación de estas compañías (tabla 2). Aunque la información disponible en el Registro Mercantil resultaba incompleta, permitía observar algunas tendencias. Por ejemplo, la poca equivalencia entre las empresas de mayor volumen de producción programada y las de mayor facturación. Así, la empresa con mayores ingresos era Linze, con más de 14 millones de $€$ en el año fiscal 2006, 
Producción independiente de televisión en Andalucía. Aproximación a la estructura de un sector desconocido

una posición mucho más alta que la séptima que ocupaba entre las productoras andaluzas más programadas. A continuación se situaban ZZJ, con más de 9,5 millones de $€$-aunque casi dos millones por debajo de su facturación en 2005- y Producciones 52, la primera por volumen de producción, con más de 8 millones de $€$. Por encima de los $2.990 .741 €$ de media que facturaron las productoras andaluzas también se encontraban Andalucía Digital Multimedia (ADM), Videoplaning y Mettre y, tomando datos del 2005, REDacción 7 Andalucía. En conjunto, el volumen de ingresos que consiguieron las diferentes productoras entre 2003 y 2006 se mantuvo en un nivel medio bastante estable, aunque en el global la facturación había aumentado en un 14,94\%, más de 7 millones de euros.

Tabla 2. Facturación de las productoras andaluzas.

\begin{tabular}{|c|c|c|c|c|c|}
\hline $\mathbf{N}^{0}$ & Productora & 2003 & 2004 & 2005 & 2006 \\
\hline 1 & Linze Televisión S.A. & 7.731 .541 & 8.354 .708 & 9.450 .764 & 14.338 .908 \\
\hline 2 & ZZJ S.A. & 4.426 .195 & 4.046 .993 & 11.392 .817 & 9.542 .294 \\
\hline 3 & Producciones 52 Andalucía S.L. & 8.335 .019 & 7.424 .660 & 7.659 .301 & 8.286 .331 \\
\hline 4 & Andalucía Digital Multimedia S.A. & 4.386 .279 & 4.070 .592 & 3.452 .439 & 4.809 .147 \\
\hline 5 & Videoplaning S.L. & 3.003 .800 & 3.448 .993 & 4.033 .783 & 3.830 .229 \\
\hline 6 & Mettre S.A. & 3.309 .545 & 3.048 .706 & 3.767 .692 & 3.453 .977 \\
\hline 7 & Red Acción 7 Andalucía S.L. & 607.854 & 3.256 .801 & - & - \\
\hline 8 & $\begin{array}{l}\text { Silencio Productora de Radio y Televisión } \\
\text { S.L. }\end{array}$ & 2.064 .821 & 2.038 .401 & 2.081 .760 & 2.872 .231 \\
\hline 9 & $\begin{array}{l}\text { Sociedad para el Desarrollo de las } \\
\text { Telecomunicaciones en Andalucía S.A. }\end{array}$ & 4.955 .792 & 5.364 .467 & 1.940 .882 & 2.516 .081 \\
\hline 10 & Estarciera S.L. & 2.202 .041 & 3.804 .475 & 2.873 .216 & 2.246 .206 \\
\hline 11 & Atrium Digital S.A. & 2.103 .489 & - & - & - \\
\hline 12 & Caligari Films S.A. & 1.967 .083 & 1.865 .458 & 1.639 .804 & 1.443 .053 \\
\hline 13 & Jondo S.L. & 1.138 .442 & - & - & - \\
\hline 14 & Mediasur Producciones Audiovisuales S.L. & - & - & 1.517 .341 & 859.593 \\
\hline 15 & $\begin{array}{l}\text { Agencia Europea de Noticias y Programas } \\
\text { TV Euro Programmes S.L. }\end{array}$ & 494.664 & 230.429 & 843.290 & 619.746 \\
\hline 16 & Tarea Sur S.A. & 842.047 & 774.688 & 662.961 & 533.478 \\
\hline 17 & Imago Video S.L. & 274.670 & - & 273.200 & 406.560 \\
\hline 18 & Producciones Zig Zag S.L. & - & - & 321.166 & 374.800 \\
\hline 19 & Savitel Productions S.A. & 616.041 & 559.998 & 393.692 & 315.106 \\
\hline 20 & Videoproducciones Al Andalus S.L. & 194.626 & 189.975 & 196.231 & 209.808 \\
\hline 21 & Digit Y Suit S.L. & 782.744 & 629.324 & 376.297 & 158.358 \\
\hline 22 & PC 29 Televisión S.A. & - & - & - & 8.166 \\
\hline- & División XL Producciones S.L. & - & - & - & - \\
\hline- & Producciones Lula S.L. & - & - & - & - \\
\hline- & Diputación de Cádiz & - & - & - & - \\
\hline & Media & 2.601 .931 & 3.069 .292 & 2.937 .591 & 2.990 .741 \\
\hline & TOTAL & 49.436 .693 & 49.108 .668 & 52.876 .636 & 56.824 .072 \\
\hline
\end{tabular}

Fuente: Elaboración propia a partir de SABI. 
Las razones de la falta de equivalencia entre las productoras que más horas colocaron en las parrillas de televisión y su nivel de facturación pueden ser de diversa índole:

1. El diferente valor del producto según la televisión de destino, el tipo de programa o la franja en que se ubica el mismo.

2. El destino de sus producciones televisivas a mercados no contemplados en la presente investigación, como los de la televisión local o internacional.

3. La diversificación de muchas de estas empresas, que no sólo producen programas para televisión sino que también se dedican a la producción cinematográfica (PC29), publicitaria (ADM, Lula Producciones, Savitel) o industrial (Savitel), así como a tareas de postproducción, producción radiofónica, multimedia (Videoplaning) o discográfica (ZZJ), gestión de derechos (Videoplaning), producción de sistemas interactivos (Videoplaning, ZZJ), alquiler de platós y equipos (Savitel), eventos corporativos (Mettre), etc.

\section{5. ¿QUIÉN COMPRA LA PRODUCCIÓN ANDALUZA?}

Toda producción televisiva independiente precisa de un canal que se encargue de su difusión. Para el conjunto de la industria andaluza, ese destino tenía un nombre claro: Canal Sur Televisión. En la temporada 2004/05, la televisión del ente público RTVA absorbió 1.962,28 horas producidas por empresas de la región, lo que representó el $87,30 \%$ de todas las horas de estas productoras (gráfico 3). En la siguiente temporada, esta cifra ya de por sí elevada ascendió todavía más hasta alcanzar las $2.009,72$ horas, un $92,16 \%$ del total de las producidas en Andalucía.

Gráfico 3. Destino de la producción independiente andaluza (\%).

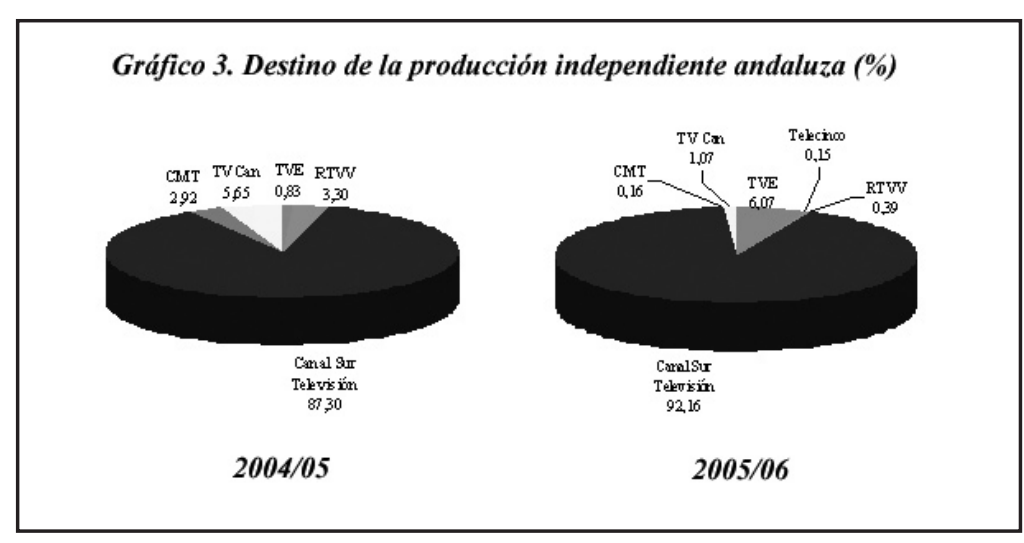

Fuente: Elaboración propia a partir de GECA y SABI. 
Más allá de los contenidos absorbidos por Canal Sur Televisión, el resto de producciones se repartió entre las televisiones autonómicas de las Islas Canarias, Castilla-La Mancha, la Comunidad Valenciana y un simbólico 0,83\% con destino a Televisión Española (TVE), la única cadena de ámbito estatal en la que se estrenó producción independiente andaluza, "Ankawa" de Videoplaning y "Gente de primera" de ZZJ. Ni un solo minuto de producción a lo largo de 2004/05 fue a parar a las televisiones privadas, de manera que la dependencia del sector público era total. En la temporada 2005/06 hubo un mayor volumen de horas con destino a las televisiones de ámbito estatal: 135,68 frente a las 18,62 de la temporada previa. Además, también aumentó el número de programas y de productoras que vendían a estas televisiones: repetían para TVE1 Videoplaning ("Ankawa") y ZZJ ("Gente de primera", "Objetivo: gente de primera" y "Eurovisión junior") y se añadían Silencio ("El loco de la colina") y Estarciera para Telecinco ("Los Morancos channel $n^{\circ} 5$ "). En total, un 6,22\% de la producción independiente andaluza.

A pesar de la ligera diversificación de la última temporada analizada, se observaba una fuerte dependencia de un único operador, Canal Sur Televisión, lo que se veía confirmado por la estructura extremadamente centralizada del ARS aplicado a las productoras independientes de la comunidad autónoma y a sus clientes (figura 1). La centralidad de los dos canales de la televisión pública regional era absoluta y todas las productoras se organizaban en forma de estrella casi perfecta en torno a ellos. En la temporada 2004/05, 23 productoras trabajaban para la televisión pública regional, 17 de ellas en exclusiva, mientras que las seis restantes tenían algún otro cliente: Producciones 52, Tarea Sur, Estarciera, ZZJ, Videoplaning y Mettre.

Figura 1. Destino de la producción independiente andaluza (2004/05, horas).

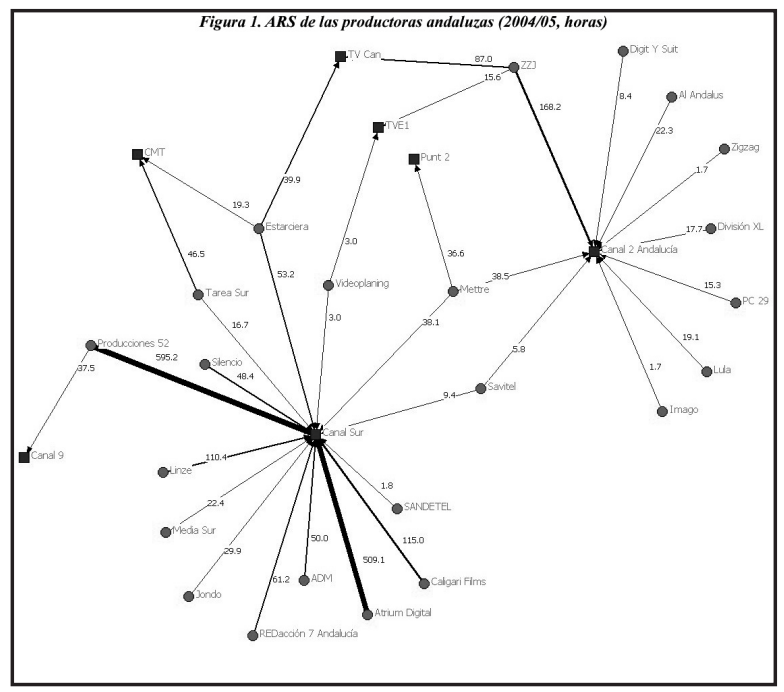

Fuente: Elaboración propia. 
En 2005/06 el sociograma de la producción independiente andaluza presentaba algunos matices con respecto a la temporada anterior (figura 2). Se continuaba apreciando una clara estrella alrededor de los dos canales de Canal Sur Televisión, con 16 productoras cuyo único cliente era la televisión pública regional. Otras tres (ZZJ, Videoplaning y Tarea Sur) también producían para ella sin exclusividad, mientras que Silencio y Estarciera sólo trabajaban con televisiones de ámbito estatal. ZZJ era la más diversificada, con cuatro clientes distintos: los dos canales de la RTVA, Televisión Canaria y Televisión Española.

Figura 2. Destino de la producción independiente andaluza (2005/06, horas).

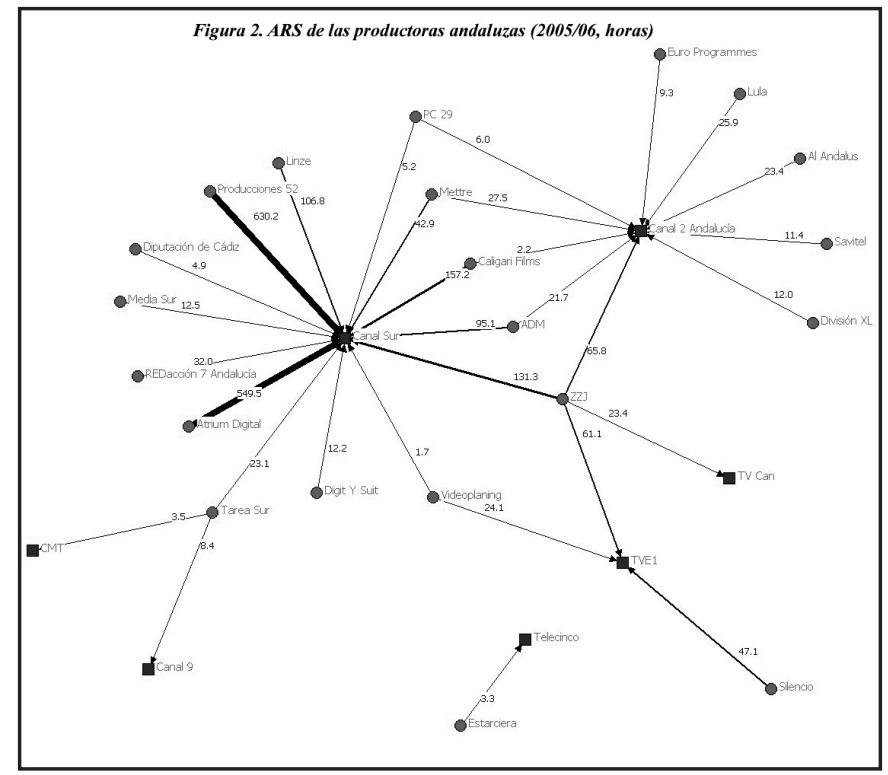

Fuente: Elaboración propia.

\section{EL PAPEL DE CANAL SUR}

La puesta en marcha de la televisión pública andaluza ha sido sin duda el factor más importante introducido en la estructura de los medios de la comunidad autónoma a lo largo de su historia. El inicio de las emisiones de Canal Sur el 28 de febrero de 1989 supuso la puesta en marcha de un potente vehículo de expresión de la cultura y la identidad andaluza, pero también introdujo los elementos necesarios para un cambio en el sistema audiovisual regional.

Creada la Radiotelevisión de Andalucía (RTVA) con la Ley 8/1987, de 9 de diciembre, de creación de la Empresa Pública de la Radio y Televisión de Andalucía y regulación de los servicios de radiodifusión y televisión gestionados 
Producción independiente de televisión en Andalucía.

Aproximación a la estructura de un sector desconocido

por la Junta de Andalucia ${ }^{30}$, su modificación con la Ley 10/2002, de 21 de diciembre, por la que se aprueban normas en materia de tributos cedidos y otras medidas tributarias, administrativas y financieras ${ }^{31}$ introdujo una nueva redacción del artículo $3^{\circ}$, en el que entre otras disposiciones establecía como deber de la RTVA "fomentar la producción audiovisual andaluza facilitando el desarrollo de la industria audiovisual". Esta obligación tiene que ver directamente, pues, con las relaciones entre las productoras audiovisuales de la comunidad autónoma andaluza y el brazo televisivo de la RTVA, Canal Sur Televisión.

La televisión pública andaluza destacaba en las temporadas analizadas por su alto volumen de contratación de producción externa. De hecho, Canal Sur, la marca de su primer canal, se encontraba en 2004/05 entre los canales españoles con mayor índice de producción independiente, con casi 1.979 horas. Sumando la externalización de Canal 2 Andalucía, durante esa temporada la RTVA compró a productoras más de 2.332 horas, sólo por detrás de la televisión pública vasca. Ello representó un $12,93 \%$ de toda la producción independiente programada en el conjunto de la televisión de ámbito estatal y autonómico en España. En 2005/06, sin embargo, RTVA redujo un $10,27 \%$ las emisiones de producción independiente hasta $2.092,85$ horas, lo que equivalía al $10,76 \%$ de las $19.455,42$ externalizadas por las televisiones estatales y autonómicas.

De ese volumen de programación, en $2004 / 05$ un $84,13 \%$ fue contratado a productoras con sede en Andalucía, ascendiendo hasta un 96,03\% en la temporada posterior (gráfico 4), de manera que la RTVA estaba ejerciendo un claro papel de motor de la industria audiovisual de la comunidad. Sólo en 2005 invirtió 45 millones de $€$ en financiar la producción independiente (Ramírez Alvarado, 2005).

Gráfico 4. Origen de la producción independiente en RTVA (\%).

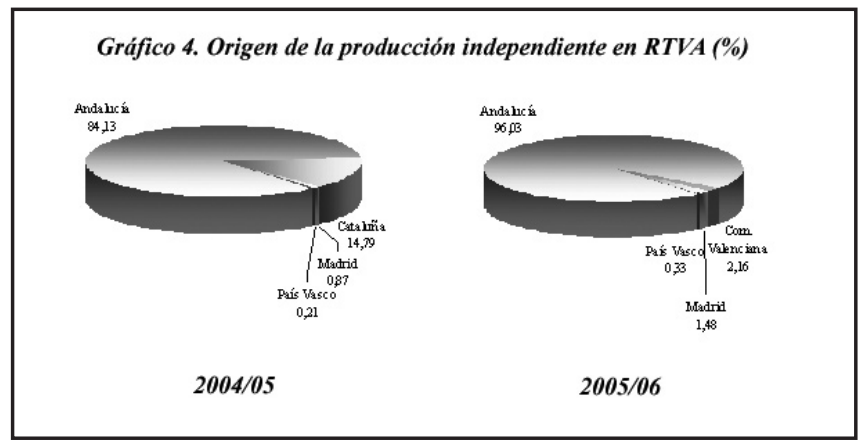

Fuente: Elaboración propia a partir de GECA y SABI.

\footnotetext{
${ }^{30}$ Boletín Oficial de la Junta de Andalucía, n 104, de 12 de diciembre de 1987.

${ }^{31}$ Boletín Oficial de la Junta de Andalucía, n 151, de 24 de diciembre de 2002.
} 
Así, en 2004/05 se detectaba una presencia relativamente importante de productoras cuya sede social estaba en Cataluña, como Mediapro (responsable de casi 300 horas en Canal Sur con "Andalucía directo"), Lua ("Generación XXI") y El Terrat ("Me lo dices o me lo cuentas"), además de madrileñas, como Cruz Delgado ("Don Quijote de la Mancha"), Tesauro ("Guadalquivir") o BRB ("Ruy el pequeño Cid"), y la vasca Pausoka ("La vida en un día"), la única que penetraba en un segundo canal convertido en bastión de las productoras locales, que en esa temporada coparon el $98,62 \%$ de su tiempo de emisión de producción independiente (figura 3).

Figura 3. Origen de la producción independiente en RTVA (2004/05, horas).

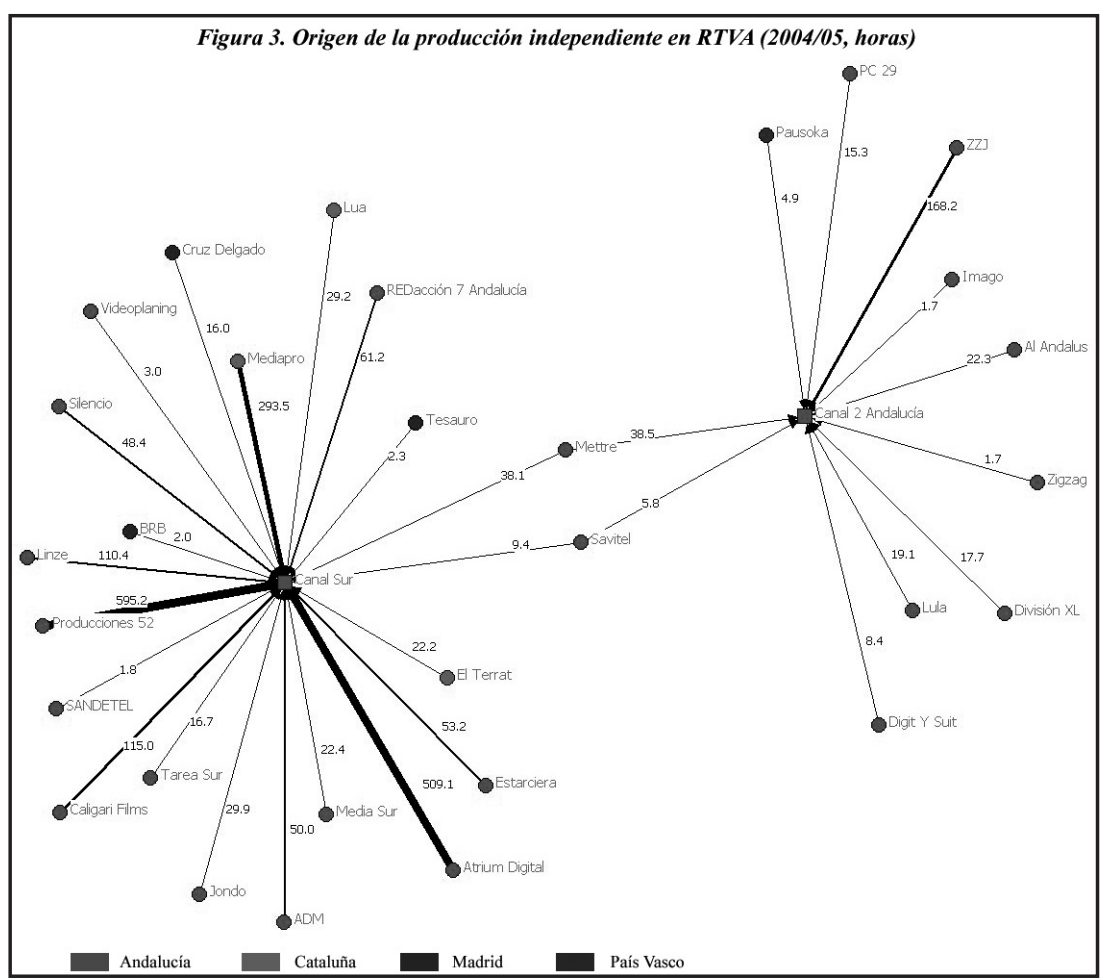

Fuente: Elaboración propia a partir de GECA y SABI.

En la siguiente temporada, el recorte en la emisión de producción independiente por parte de la RTVA tuvo su reflejo no sólo en el descenso global de la producción andaluza, como se ha indicado anteriormente, sino también en una menor presencia de productoras foráneas (figura 4). En 2005/06 repitió Pausoka 
(nuevamente con "La vida en un día") y se incorporaron a la lista de proveedores las madrileñas Prime Time ("Escúchala otra vez") y Europroducciones ("El compromiso"), así como la valenciana Extra TV ("Shalakabula").

Figura 4. Origen de la producción independiente en RTVA (2005/06).

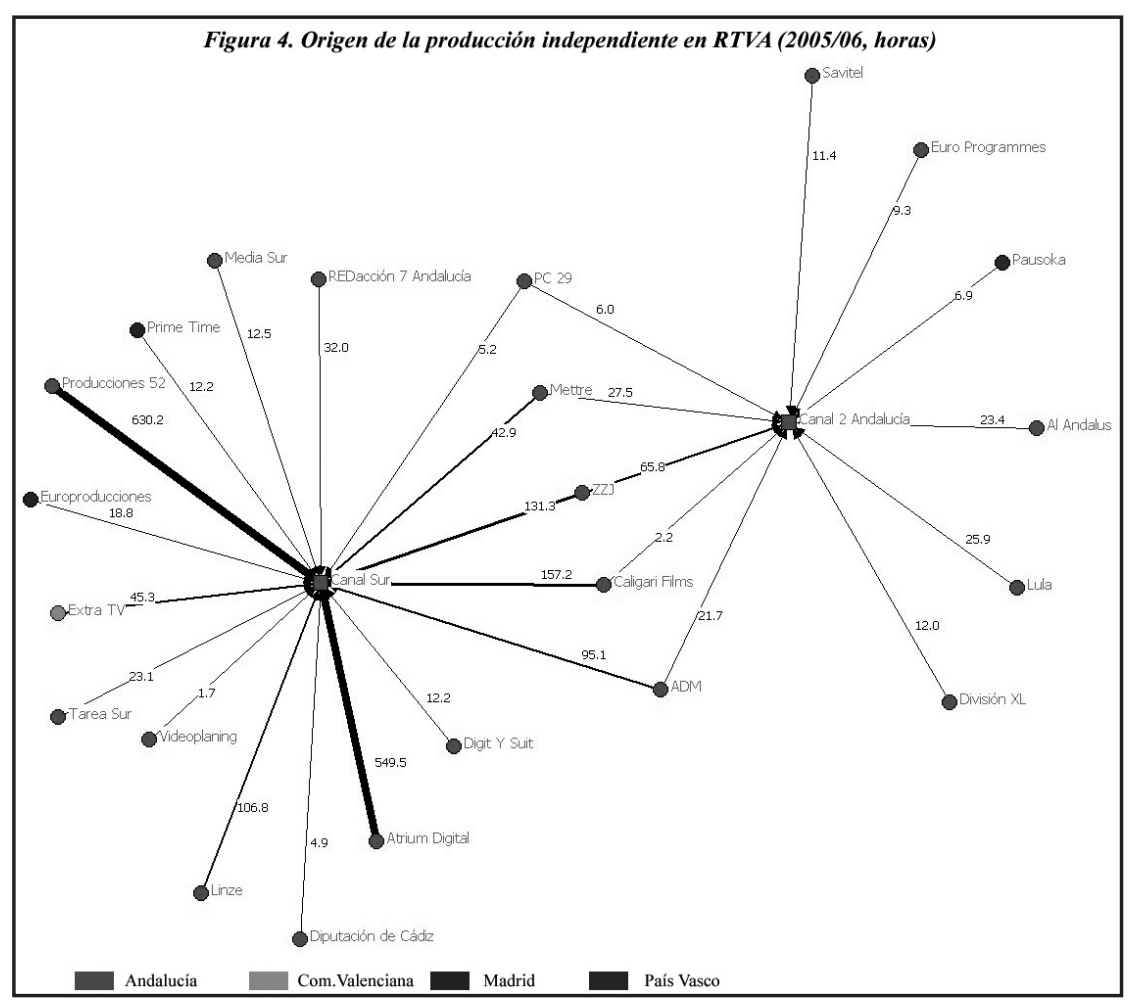

Fuente: Elaboración propia a partir de GECA y SABI.

La fuerte externalización fue una apuesta consciente de la propia televisión pública regional, ya que "en sus inicios no se dota de una maquinaria pesada basada en los modelos mastodónticos de las televisiones públicas europeas que ya habían demostrado su inoperancia en los últimos años" (Fernández Soriano, 1999: 123), de manera que, en opinión de la misma autora, "al impulso de la televisión pública, en Andalucía se ha creado una industria audiovisual que ha preparado y cualificado una mano de obra competitiva, y que ha generado un todavía débil tejido productivo" (Fernández Soriano, 1994: 159-160), a pesar de lo cual en poco tiempo la televisión incrementó su apuesta por la producción orgánica. De esta manera, se observa que el elemento central de la estructura productiva andaluza lo constituía la televisión pública autonómica. 


\section{CONCLUSIONES}

El análisis del sector de la producción audiovisual nos desvela, en primer lugar, un núcleo duro de empresas que domina el sector, tanto en volumen de producción como en facturación. No siempre son coincidentes ambas listas, y ya se han apuntado algunas de las razones, aunque en lo que sí coinciden es en su alta dependencia de Canal Sur Televisión, segunda conclusión que se desprende del análisis.

La fuerte demanda del brazo televisivo de la RTVA ha permitido crear un tejido productivo creciente en la región, en sintonía con el papel que se le ha atribuido como motor de la industria audiovisual de la región, tanto en la propia ley de este ente público como en otros documentos oficiales y en sintonía con las líneas de trabajo de otros organismos igualmente nucleares para el audiovisual andaluz, como la Fundación Audiovisual de Andalucía, en una aportación que debe ser reconocida tomando como referencia los números disponibles. No obstante, ello ha tenido a la vez el efecto perverso de aumentar la dependencia de las productoras respecto de este gran cliente, al menos en lo que hace referencia a producción televisiva, creando un mercado proteccionista. No se trata, en ningún caso, de una situación excepcional en el contexto español, en el que apenas algunas productoras catalanas, madrileñas y vascas han conseguido un modelo diversificado geográficamente (Fernández Quijada, 2007).

Esta política de apoyo público ha tenido sus efectos positivos en este período, pero en el tránsito al universo digital parece necesaria una reflexión de los actores implicados en un doble sentido.

En primer lugar, las estrategias empresariales deben buscar un universo mayor que el estrictamente andaluz. Así lo han visto ya algunas compañías y así debe entenderse la diversificación del destino de la producción emprendida por Mettre con su filial Mettre Levante, una productora ubicada en la Comunidad Valenciana que pretende reducir la dependencia respecto de la televisión pública andaluza. También ZZJ controla otra productora valenciana, Createle. Digit Y Suit, por su parte, se ha establecido en Extremadura tras las temporadas analizadas y ha empezado a trabajar con el operador público regional de esa comunidad autónoma Esta línea, además, encaja con la estrategia de internacionalización que, por ejemplo, se impulsa desde la Fundación Audiovisual de Andalucía, y encaja también en un modelo de desarrollo empresarial, el andaluz, en el que las pequeñas productoras independientes juegan un papel central, y del que de momento se mantienen relativamente alejadas las grandes productoras y los grandes grupos de comunicación de ámbito estatal, con las excepciones de Mediapro y la división española de Endemol.

En segundo lugar, la función jugada por el sector público también debe ser reinterpretada, de manera que éste abandone progresivamente un rol proteccionista que resulta extremo y contraproducente para el desarrollo de una industria auténticamente independiente, en línea con lo denunciado anteriormente para 
Producción independiente de televisión en Andalucía.

Aproximación a la estructura de un sector desconocido

este sector en España por Bustamante y Álvarez Monzoncillo (1999) o Moragas y Prado (2000). La solución pasa por la búsqueda de nuevas estrategias que faciliten la independencia real de las productoras y favorezcan su competitividad, forzando su acceso a un mercado español que con la multiplicación de canales de TDT va a precisar en los próximos años de nuevos contenidos, tanto a nivel autonómico como local y estatal, además de las posibilidades que brindan otras plataformas de transmisión como el satélite, el cable, la IPTV o los sistemas móviles. Sirva como ejemplo de que no se están aprovechando estas posibilidades el hecho de que las productoras andaluzas fueron incapaces de vender un solo minuto a los nuevos canales hertzianos generalistas Cuatro y La Sexta en su primera temporada.

Paradójicamente, si la aprobación de la Ley del Tercer Cana ${ }^{32}$ y la aparición de entes públicos de radiodifusión en diferentes comunidades autónomas contribuyeron al desarrollo de una industria audiovisual descentralizada en las regiones españolas y, entre ellas, Andalucía, hoy en día se reproduce dentro de esta misma comunidad un fuerte proceso de centralización, que puede considerarse la tercera y última conclusión del trabajo. Esta recentralización de la producción independiente concentra en la capital y en la vecina comarca del Aljarafe prácticamente el $90 \%$ de su volumen de producción, impidiendo en muchos casos el desarrollo del audiovisual en otras zonas de la región. Ni tan siquiera la parcial descentralización de la actividad de Canal Sur en las provincias, con el caso destacado del centro de producción de Málaga, ha repercutido en un desarrollo potente de este tejido en las mismas.

\section{REFERENCIAS BIBLIOGRÁFICAS}

BORGATTI, S.P.; EVERETT, M.G.; FREEMAN, L.C. (2002): Ucinet 6 for Windows: Software for Social Network Analysis. Harvard: Analytic Technologies.

BUSTAMANTE, E. (1999): La televisión económica. Financiación, estrategias y mercados. Barcelona: Gedisa.

BUSTAMANTE, E.; ÁLVAREZ MONZONCILLO, J.M.: "España: la producción audiovisual en el umbral digital". Zer, n 7 (Diciembre 1999), pp. 45-64.

CASADO, M.A.: "Nuevas estrategias para el desarrollo del sector audiovisual en las comunidades autónomas". Ámbitos, no 13-14 (2005), pp. 109-131.

CASTRO, B.; MANFREDI, J.L.; NAVARRO, J.A. (1999): RTVA, diez años con Andalucía. Sevilla: Dirección de Imagen y Comunicación RTVA.

FERNÁNDEZ-QUIJADA, D.: "El mercado de producción independiente en España ante la aparición de Cuatro y laSexta". Comunicación y sociedad, vol. 22 (Junio 2009), n 1 [en prensa].

\footnotetext{
${ }^{32}$ Ley 46/1983, de 26 de diciembre, reguladora del Tercer Canal de Televisión (Boletín Oficial del Estado, $\mathrm{n}^{\circ} 4$, de 5 de enero de 1984).
} 
-: El análisis de redes sociales aplicado al estudio de la estructura de las industrias culturales. En (2008): Actas y Memoria Final. Congreso Internacional Fundacional AEIC. Santiago de Compostela: Asociación Española de Investigación de la Comunicación. [Consulta: 20 abril 2008]. <http://www.griss.org/curriculums/fernandezquijada/publicaciones/aeic fernandezquijada.pdf>.

- (2007): Las industrias culturales ante el cambio digital. Propuesta metodológica y análisis de caso de la televisión en España. Tesis doctoral. Bellaterra: Universitat Autònoma de Barcelona. [Consulta: 24 abril 2008]. <http://www.tesisenxarxa.net/TESIS UAB/AVAILABLE/ TDX-0523108-151341//dfq1de1.pdf>.

FERNÁNDEZ SORIANO, E.: La televisión andaluza: un instrumento público para la integración. En DÍAZ-NOSTY, B. (dir.) (1994): Comunicación social 1994/Tendencias. Madrid: Fundesco, pp. 159-160.

-: El desarrollo del sector de la producción audiovisual en Andalucía. En AGUILERA, M.; MENDIZ, A.; CASTILLO, A. (coords.) (1999): La industria audiovisual y publicitaria en Andalucía. Estudios sobre un sector estratégico. Málaga: Universidad de Málaga, pp. 117-138.

GECA (2006): El anuario de la televisión 2006. Madrid: Gabinete de Estudios de la Comunicación Audiovisual.

- (2007): El anuario de la televisión 2005/06. Madrid: Gabinete de Estudios de la Comunicación Audiovisual.

MARTÍNEZ, M.: 'Arrayán' echa raíces. Sur.es [en línea]. 25 noviembre 2007 [Consulta: 27 abril 2008]. <http://www.diariosur.es/20071125/sociedad/arrayan-echa-raices-20071125. html>.

MORAGAS, M.; PRADO, E. (2000): La televisió pública a l'era digital. Barcelona: Pòrtic.

RAMÍREZ ALVARADO, M. (coord.) (2005): Guía del audiovisual en Andalucía 2005-2006. Producción de cine y televisión. Sevilla: Fundación Audiovisual de Andalucía.

ZALLO, R.: Políticas culturales territoriales: una experiencia rica pero insuficiente. En BUSTAMANTE, E. (coord.) (2002): Comunicación y cultura en la era digital. Industria, mercados y diversidad en España. Barcelona: Gedisa, pp. 277-305.

\section{Breve semblanza biográfica del autor}

David Fernández-Quijada es Profesor ayudante doctor del Departamento de Comunicación Audiovisual y de Publicidad de la Universitat Autònoma de Barcelona, en la que se doctoró. Sus áreas de investigación son la economía de las industrias culturales en el entorno digital, el servicio público de radiodifusión y las nuevas tecnologías de la comunicación.

(Recibido el 06-05-2008, aceptado el 01-03-2009) 